\title{
Population structure in the MHC region
}

André Silva Maróstica ${ }^{1}$, Kelly Nunes ${ }^{1}$, Erick C. Castelli ${ }^{2,3}$, Nayane S. B. Silva ${ }^{3}$, Bruce S. Weir ${ }^{4}$, Jérôme Goudet ${ }^{5}$, Diogo Meyer ${ }^{1}$.

1. Universidade de São Paulo, Departamento de Genética e Biologia Evolutiva, São Paulo, SP, Brazil.

2. Universidade Estadual Paulista - Unesp, Faculdade de Medicina de Botucatu, Departamento de Patologia, Botucatu, SP, Brazil.

3. Molecular Genetics and Bioinformatics Laboratory, Experimental Research Unit, School of Medicine, São Paulo State University - Unesp, Botucatu, SP, Brazil.

4. Department of Biostatistics, University of Washington, Seattle, Washington 98195.

5. Department of Ecology and Evolution,Swiss Institute of Bioinformatics, University of Lausanne, 1015 Switzerland. 


\section{Abstract}

In his 1972 "The apportionment of human diversity", Lewontin showed that, when averaged over loci, genetic diversity is predominantly attributable to differences among individuals within populations. However, selection on specific genes and genomic regions can alter the apportionment of diversity. We examine genetic diversity at the HLA loci, located within the MHC region. HLA genes code for proteins that are critical to adaptive immunity and are well-documented targets of balancing selection. The SNPs within HLA genes show strong signatures of balancing selection on large timescales and are broadly shared among populations, with low $F_{S T}$ values. However, when we analyze haplotypes defined by these SNPs (i.e., which define "HLA alleles"), we find marked differences in frequencies between geographic regions. These differences are not reflected in the $F_{S T}$ values because of the extreme polymorphism at $\mathrm{HLA}$ loci, illustrating challenges in interpreting $\mathrm{F}_{\mathrm{ST}}$. Differences in the frequency of HLA alleles among geographic regions are relevant to bone-marrow transplantation, which requires genetic identity at HLA loci between patient and donor. We explore the case of Brazil's bone-marrow registry, where a deficit of enrolled volunteers with African ancestry reduces the chance of finding donors for individuals with an MHC region of African ancestry.

Keywords: Population structure, MHC, HLA genes, transplantation, population-specific $\mathrm{F}_{\mathrm{ST}}$ 


\section{Introduction}

In "The apportionment of human genetic diversity", Richard Lewontin addressed a well-defined and answerable question: "how much of human diversity between populations is accounted for by more or less conventional racial classification?" With the genetic data available at the time and drawing on existing classifications of human races, he reached the unequivocal result that individuals assigned to different races are, on average, only slightly more genetically different than those from the same race.

Lewontin's work redefined our way of thinking about, and referring to, human genetic variation, bringing an awareness that racial labels are arbitrary from the biological perspective, and largely socially constructed. Appropriately, subsequent studies shifted the focus to an understanding of how human genetic variation is distributed across the globe, what are the geographic scales at which such variation is observed (Rosenberg et al. 2005), and which evolutionary processes account for the observed patterns (Manica, Prugnolle, and Balloux 2005; Prugnolle, Manica, and Balloux 2005). What Lewontin referred to as "apportionment" has largely been recast in terms of "population structure", and his approach to describing variation is now explored using metrics related to population genetic models, as is the case of studies using $\mathrm{F}_{\mathrm{ST}}$.

Lewontin was aware that his main result referred to an "average" behavior over loci, and that variation in population structure among loci arises as a consequence of evolutionary sampling, as well as the locus-specific effects of natural selection. He explored this idea in (Lewontin and Krakauer 1973) by using the properties of the observed distribution of $\mathrm{F}_{\mathrm{ST}}$ over loci to make inferences about natural selection. While this effort had limited success due to the difficulty in defining an appropriate null expectation for the distribution of $F_{\mathrm{ST}}$ (Nei and Maruyama 1975), the strategy has since become a central approach to study natural selection. In the genomic era, outlier approaches can be used (i.e., the detection of loci with extreme $F_{S T}$ ), and the sheer scale of the data also allows parameter-rich models to be used to produce simulations that provide a null model (Nielsen 2005).

Here, we discuss the apportionment of genetic diversity for a particular set of loci, the HLA genes, which are located on chromosome 6 in a region of approximately 4 megabase known as the Major Histocompatibility Complex (MHC). The HLA genes have long attracted the interest of evolutionary biologists because of their unusually high levels of polymorphism (Bodmer 1972). The function of the proteins coded by HLA genes helps understand this unusual diversity: HLA proteins bind peptides and present them on the cell surface to T-cell receptors,

Population Structure in the MHC, Meyer et al. 
triggering a cellular or humoral immune response if the MHC-peptide complex is recognized as foreign, as is the case for peptides from pathogens (Bodmer 1972; Klein and Sato 2000). The repertoire of peptides an individual can present on the cell surface depends on the HLA alleles which are present. As a consequence, pathogen diversity engages in a coevolutionary interaction with HLA loci. This interaction explains why HLA genes stand out as having the strongest evidence of balancing selection in the genome (Meyer et al. 2018), with (a) elevated non-synonymous substitution rates (Hughes and Nei 1992), (b) alleles with frequencies that deviate from neutral expectations (Hedrick and Thomson 1983), (c) DNA sequences with an excess of common variants (Bitarello et al. 2018), and (d) far more polymorphisms that are shared among species than expected under neutrality (Leffler et al. 2013).

Here, we investigate how selection affects the apportionment of genetic variation at HLA loci. The population structure at HLA loci is informative about the mode and timescale of selection, and the prevalence of local adaptation (e.g., selection favoring variants in a specific region) versus geographically widespread selection (e.g., selection favoring polymorphism which is shared among distantly related populations). An understanding of population structure at HLA loci is also relevant to Hematopoietic Stem Cell Transplantation (HSCT, also known as bone marrow transplantation), an important curative procedure used in the treatment of various forms of cancer and hematological diseases, where cells capable of generating hematopoietic tissue are transferred from a donor to a recipient. Successful HSCT requires genetic identity at HLA loci between patient and donor, a condition which is related to how genetic variation is apportioned. We will explore the relevance of population structure at HLA loci to challenges in planning the recruitment of unrelated donors for HSCT in Brazil, a country with a highly admixed population, with large components of European, African and Native American ancestry (Salzano and Bortolini 2005; Souza et al. 2019).

\section{Data analysis}

The 1000 genomes data. For our reanalysis of population structure in the MHC region, we have chosen the data for 20 non-admixed populations from Phase 3 of the 1000 Genomes Dataset (hereafter 1000G) (1000 Genomes Project Consortium and Adam Auton, Lisa D Brooks, Richard M Durbin, Erik P Garrison, Hyun Min Kang, Jan O Korbel, Jonathan L Marchini, Shane McCarthy, Gil A McVean, Gonçalo R Abecasis 2015). The $1000 \mathrm{G}$ data allows us to compare variation of the $\mathrm{MHC}$ to that in other genomic regions, to make calls for nucleotide positions within HLA loci, and to estimate which HLA alleles an individual carries (as described 
later). By "HLA alleles" we refer to the phased combination of variants within a locus, an important unit of analysis since it is the HLA alleles that define immunological phenotypes which contribute to disease and adaptation. Although the sampling structure of the $1000 \mathrm{G}$ is much coarser than that used by Lewontin (1972), we will be able to compare genetic variation among four major geographic regions (Africa, Europe, South Asia, East Asia).

HLA allele and SNP calls. Genotype calls within HLA loci for the 1000 Genomes data are biased towards higher frequencies of reference alleles, due to mapping bias (Brandt et al. 2015). We therefore made new calls by extracting reads that either map to the MHC region or are unmapped (using the 1000 Genomes release describe by (Byrska-Bishop et al. 2021) and then performing a locus-specific mapping using known HLA alleles as references (Castelli et al. 2018). Throughout the text, we refer both to analyses for SNPs within HLA genes (in our case, the $H L A-A,-B$, and $-C$ ) and the MHC region (the broader region within which these and other genes of immunological function are contained). We also analyze diversity for "HLA alleles", the phased combination of SNPs that defines an unique HLA protein sequence. Given our interest in functional variation at the HLA loci, we will analyze diversity among HLA alleles that correspond to unique protein sequences.

$\mathrm{F}_{\mathrm{ST}}$ estimates. In addition to revisiting Lewontin's approach to quantifying the apportionment of genetic variation, we estimate $\mathrm{F}_{\mathrm{ST}}$ to describe population structure. Our analyses will use the framework of Weir and Goudet (2017), where population-specific $F_{\mathrm{ST}}$ is computed based on allelic sharing and interpreted as a measure of relative kinship.

For genotypic data, for population $i$, we define the population-specific $\mathrm{F}_{\mathrm{ST}}$ metric as:

$F_{S T}^{i}=\frac{S_{w}^{i}-S_{b}}{1-S_{b}}$

$S$ refers to allele sharing between two individuals. For a bi-allelic locus, $S$ assumes a value of one if the two individuals are homozygous for the same allele, zero if they are homozygous for different alleles, and 1/2 otherwise. Multiallelic loci can be accommodated in this framework by recoding them as a series biallelic markers. $S_{w}^{i}$ is sharing within population $i$, and $S_{b}$ is sharing between pairs of populations, averaged over pairs. Population-specific $\mathrm{F}_{\mathrm{ST}}$ estimates the 
probability that two alleles drawn from population i are identical by descent (ibd) relative to a pair of alleles drawn from different populations. Averaging $F_{S T}^{i}$ over populations provides the classically defined "overall $\mathrm{F}_{\mathrm{ST}}$ " (B. S. Weir and Clark Cockerham 1984). When estimating $\mathrm{F}_{\mathrm{ST}}$ for a genomic region containing multiple SNPs, we use the "ratio of averages" approach, where the average value of the denominator and numerator are computed separately over SNPs, providing an unbiased estimator of $\mathrm{F}_{\mathrm{ST}}$ (Ochoa and Storey 2021).

The $\mathrm{F}_{\mathrm{ST}}$ estimates were computed with the Hierfstat $\mathrm{R}$ package (Goudet 2005). For the analysis of the entire chromosome, we filtered for biallelic SNPs and indels using VCFtools v.0.1.15 (Danecek et al. 2011) and converted from genotypic data to dosage format using Plink v.1.9 (Chang et al. 2015). In analyses of HLA alleles and SNPs within HLA loci, we used the genind2hierfstat and loci2genind functions (from the Hierfstat and Pegas packages) to format the data, and the fstat2dos and fs.dosage functions (from the Hierfstat package) to convert genotypic data to dosage and then calculate $\mathrm{F}_{\mathrm{ST}}$.

\section{How is HLA diversity apportioned?}

If Lewontin had HLA data available at the time of his study, what patterns would he have found? Lewontin's approach consisted in comparing genetic diversity for progressively more inclusive groups (in his case, ranging from populations, to anthropologically defined races, to the entire species). He argued that when there are large differences between groups but little variability within them, measures of diversity for the species as a whole would be higher than those for each separate group. Using his approach, we find that heterozygosity at classical HLA class I loci ( $H L A-A, H L A-B$, and $H L A-C)$ is on average only slightly higher within regions or for the entire world ("total"), as compared to within populations (Table 1).

Table 1. Diversity at the HLA allele level and for the set of SNPs within HLA genes in the 1000 Genomes data, for $H L A-A,-B,-C$. Values refer to average heterozygosities over each form of grouping individuals (populations, geographic regions, total; $\mathrm{H}_{\mathrm{p}}, \mathrm{H}_{\mathrm{r}}, \mathrm{H}_{\mathrm{t}}$, respectively). Total refers to the diversity present if all populations are treated as a single group. The regions (and populations) are: Africa (Gambian Mandinka, Mende, Esan, Yoruba, Luhya); Europe (Finnish, Iberian, Toscani, CEU - Northern Europeans, British); East Asia (Southern Han Chinese, Kinh Vietnamese, Japanese, Han Chinese, Dai Chinese); and South Asia (Bengali, Punjabi, Gujarati, Tamil, Telugu). 


\begin{tabular}{|l|c|c|}
\hline & HLA allele level Heterozygosity & SNP level Heterozygosity \\
\hline Populations & 0.904 & 0.170 \\
\hline Regions & 0.916 & 0.172 \\
\hline Total & 0.948 & 0.176 \\
\hline
\end{tabular}

In Lewontin's framework, the apportionment of HLA variation was computed as the relative gain in diversity obtained by amalgamating samples at higher level of grouping. Thus, the fraction of variation contributed by various levels of population structure can be computed for HLA allele level diversity as follows:

Within populations: $\frac{H_{p}}{H_{t}}=0.953$

Between populations within regions: $\frac{\left(H_{r}-H_{p}\right)}{H_{t}}=0.013$

Between regions: $\frac{\left(H_{t}-H_{r}\right)}{H_{t}}=0.034$

For SNP level heterozygosities, these same calculations result in $0.965,0.012,0.022$ of the total variation being due to differences within populations, between populations within regions, and between regions, respectively.

Clearly, the vast majority of HLA variation, both at the HLA allele and SNP levels, lies within populations, and little additional variation is accrued by examining higher grouping. Notice that this does not imply that there are few differences between populations and geographic regions, but rather that they add very little diversity relative to that which is already present within populations. This overall pattern has been found in other studies of HLA variation across populations, which used hierarchical $\mathrm{F}_{\mathrm{ST}}$ approaches, and also accounted for the level of divergence among HLA alleles ((Buhler and Sanchez-Mazas 2011; Sanchez-Mazas 2007; Meyer et al. 2006)).

While the overall picture of HLA variation is clearly one of high diversity within individual populations, analyses restricted to HLA genes are unable to address important questions. First, inferences about population structure of a specific locus requires placing it in a genome-wide context. This is difficult to accomplish when we use variation for entities such as "HLA genes" 
since the size of the locus, the density of SNPs, and the mutational mechanism all confound the interpretation of variation and, therefore, of the pattern of population structure. Secondly, natural selection may act heterogeneously across populations, so selective episodes that are specific to a population or region would be unnoticed in a survey of "overall" patterns of population structure, averaged over the entire dataset. This issue calls for approaches examining the evolutionary history of individual populations or lineages (Bruce S. Weir et al. 2005; B. S. Weir and Hill 2002; Bruce S. Weir and Goudet 2017), and defining a marker type that can be compared among genomic regions.

An objective way to compare the apportionment of variation at HLA genes with genome-wide values is to contrast estimates for SNPs within the MHC region, and within HLA genes, to genome-wide values (thus controlling for marker types) and using the same set of populations and individuals (thus controlling for population and individual sampling effects). In addition, using the population-specific $F_{\text {ST }}$ approach, inferences about specific populations or regions (as opposed to overall values, averaged over all populations) can be made. Here, we use the 1000 Genomes data and the population-specific $F_{S T}$ framework to revisit the question of how HLA diversity is apportioned by focusing on contrasts between SNPs in the MHC region, within HLA genes, and in the remainder of chromosome 6.

\section{MHC diversity in a genomewide context}

To visualize how population structure in the $\mathrm{MHC}$ region compares to genomewide values, we estimated the population-specific $\mathrm{F}_{\mathrm{ST}}$ for $5 \mathrm{Mb}$ windows along chromosome 6 (Figure 1). The five African populations have lower population-specific $F_{S T}$ throughout the entirety of chromosome 6, reflecting higher diversity and thus lower within-population kinship among individuals, as compared to kinship within populations from other regions. However, within the $\mathrm{MHC}$ region Africans have a higher population-specific $\mathrm{F}_{\mathrm{ST}}$ than in the remainder of the chromosome, and Asian and European populations show the opposite, i.e., a reduction in their $F_{S T}$ values within the MHC (Figure 1, see MHC region delimited by vertical lines). The increase seen for $\mathrm{F}_{\mathrm{ST}}$ in the African $\mathrm{MHC}$ region expresses the fact that, for this genomic region, Africans do not have a markedly higher diversity (and thus lower degree of within population kinship) than do other populations.

The overall $F_{S T}$ (i.e., the average of population-specific $F_{S T}$ values), reaches its lowest value in chromosome 6 within the MHC (black line in Figure 1). The outlier status of the windows in the $\mathrm{MHC}$ region can be visualized by comparing the distribution of their $\mathrm{F}_{\mathrm{ST}}$ values to

Population Structure in the MHC, Meyer et al. 
that of windows in the remainder of chromosome 6 (Figure 2, top panel). The overall $F_{\mathrm{ST}}$ for the set of SNPs contained within the three HLA genes (a subset of the MHC region) is even more extreme (Figure 2A, vertical arrow). The apportionment of diversity among regions can also be computed by treating all the populations in a geographic region as a single group and computing an overall $F_{S T}$ among regions (Figure 2, bottom panel). Once again, the $F_{S T}$ values for SNPs within the MHC and HLA genes occupy an extreme position, indicating unusually low $\mathrm{F}_{\mathrm{ST}}$ with respect to the remainder of chromosome 6 .

The outlier status of the MHC region SNPs, particularly those contained within HLA genes, supports a role for natural selection favoring the maintenance of diversity and resulting in extensive sharing of polymorphisms among regions and populations.

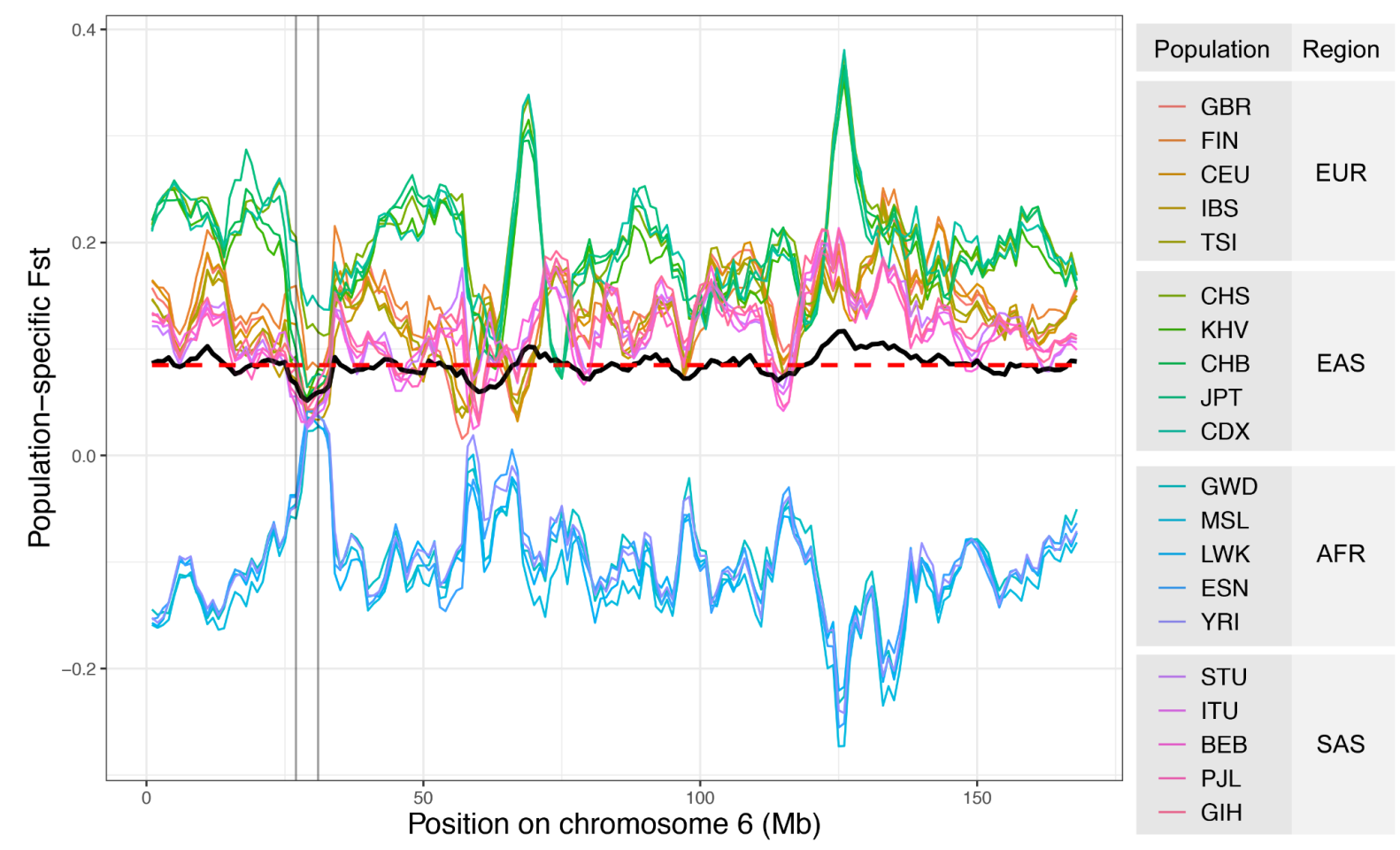

Figure 1. Population-specific $\mathrm{F}_{\mathrm{ST}}$ across chromosome 6 , in windows of $5 \mathrm{Mb}$, and step-size of $1 \mathrm{Mb}$. Each colored line represents a specific population; the black line represents the overall $F_{S T}$, for each window; the red dashed line is the average overall $F_{S T}$ for the entire chromosome. The vertical lines delimit the MHC region, within which HLA genes are contained. See supplementary Table S1 for $1000 \mathrm{G}$ abbreviations of population and region names. 

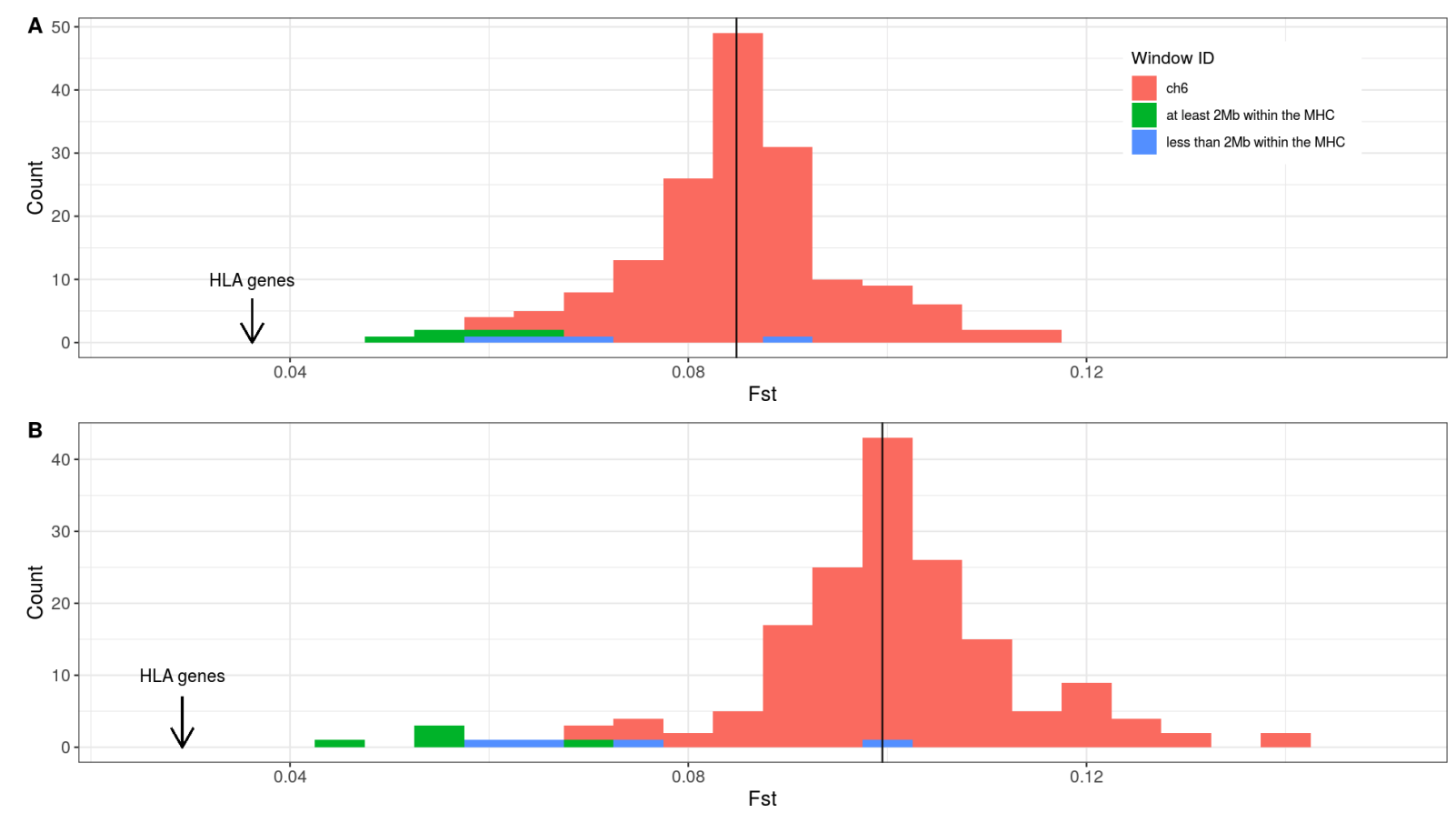

Figure 2. Distribution of overall $F_{S T}$ for 168 windows along chromosome 6 . The $F_{S T}$ values for windows that have at least $2 \mathrm{Mb}$ contained within the $\mathrm{MHC}$ are shown in green, and the windows that intersect less than $2 \mathrm{Mb}$ of $\mathrm{MHC}$ are shown in blue, while the windows completely outside the $\mathrm{MHC}$ region are in red. The arrow indicates the overall $\mathrm{F}_{\mathrm{ST}}$ for the SNPs contained within the three HLA loci we studied (HLA-A, $-B,-C$ ), and the vertical line is the average $F_{S T}$ over all windows. $(A)$ overall $F_{S T}$ among populations. (B) overall $F_{S T}$ among continents.

\section{Local adaptation at HLA loci}

The overall $\mathrm{F}_{\mathrm{ST}}$ for a locus captures the influence of evolutionary sampling and natural selection, averaged over populations. However, it is possible that selection acts in a population-specific manner, with the overall pattern masking signatures within specific populations (Bruce S. Weir et al. 2005). This has proved a relevant issue for studies of MHC diversity, which have recently found examples of specific populations with signatures of increased $F_{S T}$, in the opposite direction to the overall $F_{S T}$ values presented above.

For a set of closely related African populations (African-American, Nigerians and Gambians), Bhatia and coworkers (Bhatia et al. 2011) found that the $F_{S T}$ in the MHC region 
significantly exceeded that of the rest of the genome. For another set of African populations (Patin et al. 2017) also found an excess differentiation at the MHC region for a sample of Bantu speakers, relative to genomewide. In an analysis of Native Americans, (Nunes et al. 2021) used microsatellites within the $\mathrm{MHC}$ and spread throughout the genome to show that $\mathrm{F}_{\mathrm{ST}}$ in the $\mathrm{MHC}$ exceeded neutral expectations (obtained by simulations). Finally, (Brandt et al. 2018) found that pairwise contrasts between East Asian populations from the $1000 \mathrm{G}$ showed unusually high $\mathrm{F}_{\mathrm{ST}}$, when compared to genome-wide averages.

Local adaptation of HLA alleles is also supported by the study of admixed populations, with several studies documenting an excess of African ancestry within the MHC region (Norris et al. 2021; Meyer et al. 2018), consistent with the hypothesis that alleles of African ancestry were advantageous in the admixed populations. A similar signature was also found for the Native American component in admixed Mexicans (Zhou, Zhao, and Guan 2016), again indicating that a subset of locally advantageous alleles rose in frequency after the onset of admixture.

Thus, while there is strong evidence of reduced overall $\mathrm{F}_{\mathrm{ST}}$ for SNPs in the MHC region and HLA genes (Figures 1 and 2), studies that queried admixed or closely related sets of populations identified instances where $\mathrm{F}_{\mathrm{ST}}$ for the $\mathrm{MHC}$ region is in fact higher, and not lower, than genome-wide averages. These findings are consistent with selection favoring locally adapted variants, and emphasizes the importance of considering that selective regimes at HLA loci may differ, depending on the timescale being considered (Garrigan and Hedrick 2003).

\section{HLA diversity and transplantation}

Patients suffering from hematological diseases, including forms of cancer, can be treated with stem cells harvested from the bone marrow of a donor in a process called Hematopoietic Stem Cell Transplantation (HSCT). The ideal setting for this procedure is for donor and recipient to match at $5 \mathrm{HLA}$ loci (HLA-A, $-B,-C,-D R B 1,-D Q B 1$, referred to as 10/10 matching), with "matching" defined by identity at the protein level for HLA alleles. However, because the expected heterozygosities at the HLA allele level are typically greater than 0.90 within populations (Table 1), the chance that two unrelated individuals will match at five loci is extremely low. Therefore, the first option for patients is to search for compatible donors among close relatives. However, fewer than $30 \%$ of patients find a compatible donor among relatives, and their next option is to seek donors in registries.

An important question for registries is whether the genetic ancestry of the patient and the donor influences the chance of a match. The population structure of HLA loci is potentially 
informative about this question. For the two most common ancestries in Brazil (European and African), we computed the population-specific and overall $F_{S T}$ for populations in the 1000G, and found values to be very low (Figure 3, overall $F_{S T}=0.03$ at the HLA allele level). At first sight, this appears to indicate that there is an extensive sharing of alleles among Africans and Europeans. However, for extremely polymorphic loci, as is the case for HLA, low $F_{S T}$ is possible even in the absence of extensive sharing of alleles among populations (Hedrick 2005; Balloux et al. 2000), making it misleading to associate low $F_{\mathrm{ST}}$ to lack of differentiation.

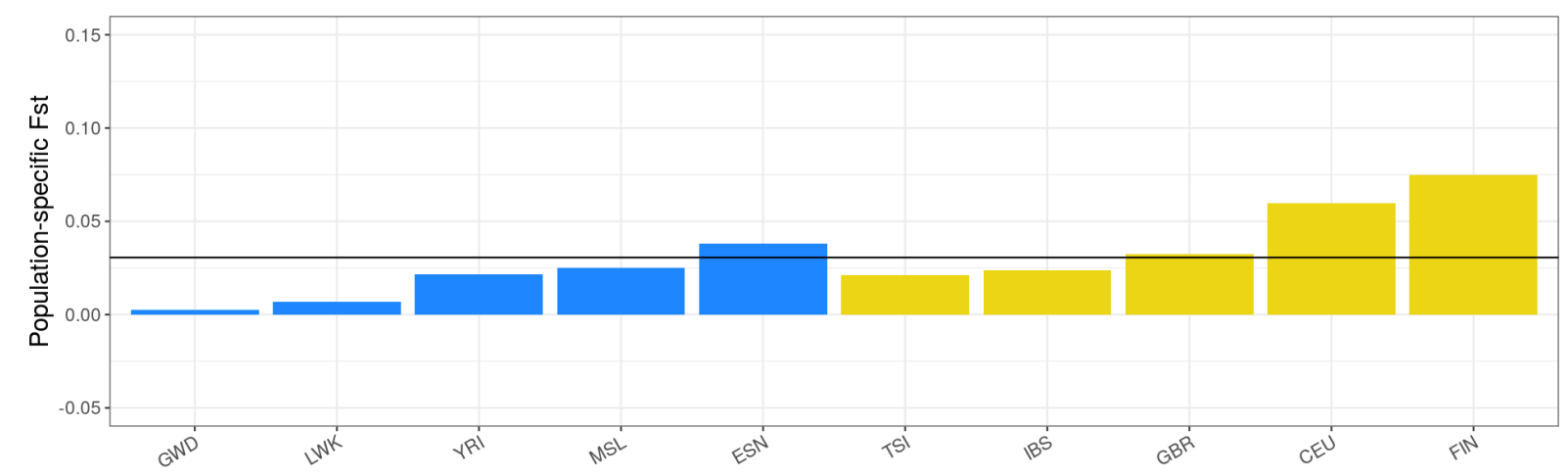

Figure 3. The population-specific $F_{S T}$ values for African (in blue) and European (in yellow) populations in the 1000G, estimated for diversity at the level of HLA alleles for HLA-A, -B, -C. The horizontal line is the average over population-specific $F_{S T}$, with a value of 0.03 .

To visualize the differences in allele frequencies between African and European populations, we identified a set of HLA alleles that are either exclusive or at least three-fold more common in Africa, as compared to Europe, in the $1000 \mathrm{G}$ data. Figure 4 illustrates how 18 HLA-A and 20 -B alleles, which collectively contribute to approximately $60 \%$ cumulative frequency in Africa, reach less than $10 \%$ in Europe (for HLA-C alleles the differences among regions are smaller). The striking differences in allele frequencies among regions for HLA-A and $-B$ are also observed in an independent dataset containing data from multiple populations (Solberg, O. D., Mack, S. J., Lancaster, A. K., Single, R. M., Tsai, Y., Sanchez-Mazas, A., \& Thomson, G. 2008) Supplementary Figure S1-S3). This analysis shows how the distribution of HLA alleles is in fact geographically structured, but as a consequence of the extreme polymorphism, the overall $F_{S T}$ is low (Hedrick 2005; Balloux et al. 2000). We do not see this as a limitation of $F_{\text {ST }}$ (as do others, e.g., (Hedrick 2005; Jost 2008)), but as an expression of the information $\mathrm{F}_{\mathrm{ST}}$ conveys, which is related to the evolutionary history of the population and locus

Population Structure in the MHC, Meyer et al. 
of interest, rather than serving as a direct measure of differences in allele frequencies. Here, low $F_{S T}$ reflects the high diversity (and low kinship) both within and between regions, a key feature of HLA polymorphism.

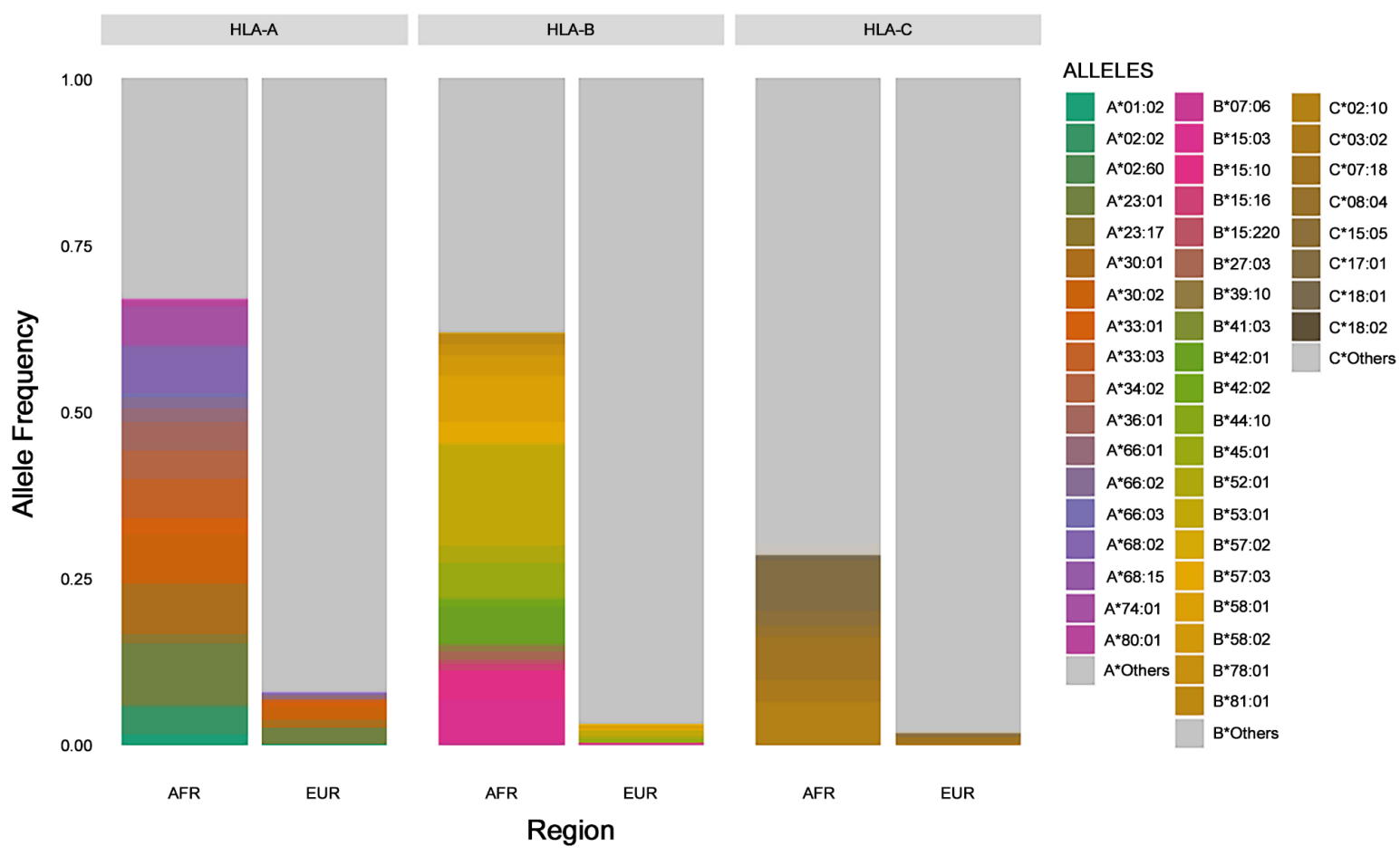

Figure 4. Frequencies of a subset of HLA alleles, for African and European populations (1000G). To visualize the differences in allele frequencies between regions, we selected alleles that were either exclusive to Africa, or found at frequencies that were at least threefold greater in Africa with respect to Europe. We identify each allele by a distinct color. "Others" (in gray) refers to the cumulative frequency of all alleles that are either exclusively found in Europe, or that do not occur threefold more frequently in Africa than Europe, or that are shared between them.

What are the consequences of the large differences in allele frequencies between geographic regions (Figure 4) to the chances of finding a matching donor in a repository? Brazil is a highly admixed country, with the largest population descended from Africans outside Africa (Ade Ajayi 1998), and a history of extensive migration from Europe. There is also an important but lower proportion of Native American ancestry, in particular in the north of the country. In Nunes et al (2020), we therefore quantified how an individual's ancestry influences their chances of finding a matching donor in Brazil's Redome, the Brazilian Bone Marrow Donor Registry. In a survey of over 8,000 admixed individuals, we showed that those who self-identify 
as "Black" have a $57 \%$ reduction in their chances of finding a matching donor, with respect to those who self-identify as "White". When dividing the cohort into groups based on genomewide ancestry, there is a $60 \%$ reduction in chances of finding a donor in a group of 1589 individuals with the greatest African ancestry, with respect to those with the least African ancestry. Finally, when the question is recast with respect to genetic ancestry specific to the $\mathrm{MHC}$ region, we found that individuals with two MHC regions with African ancestry have a $75 \%$ reduction in chances of finding a donor (Figure 5A), with respect to those with two $\mathrm{MHC}$ regions identified as European.

The lower chances of finding a donor for individuals with African ancestry genomewide (and in particular for the MHC) is a consequence of REDOME's underrepresentation of individuals of African ancestry, as documented in their statistics for self-identified labels used by the Brazilian Institute of Geography and Statistics (IBGE). Whereas the categories "Black" and "Mixed" correspond to $53.9 \%$ of Brazilians, they only make up $30.6 \%$ of registered donors in REDOME. Similar results were found for the National Marrow Donor Program (NMDP), where individuals who self-identify as "Black" have a 5-fold lower chance of finding a donor, compared to those who identify as "White" (Gragert et al. 2014).

Would increasing the proportion of individuals with African ancestry in the registries increase the chances of patients with a greater African ancestry in finding a compatible donor? Nunes et al (2020) found that individuals with a higher proportion of African ancestry in the MHC on average find proportionally more donors identified as "Black" or "Mixed" (Figure 5B). This suggests that for individuals with greater African ancestry, an increase in the proportion of donors with African ancestry will contribute to their chances of finding a compatible donor.

It may seem surprising to refer to "Black" and "Mixed" within a study that celebrates the very paper in which Lewontin provided a categorical rejection of racial categories. We believe reference to these categories is necessary in the context of the questions we address here, since government institutions routinely still employ these categories. It is appropriate to investigate their bearing on issues of public health, including the possible outcome of refuting their utility. Our results show that, in the case of finding matching donors, it is ultimately the genetic ancestry of the MHC which most strongly defines the chances of finding a match. 

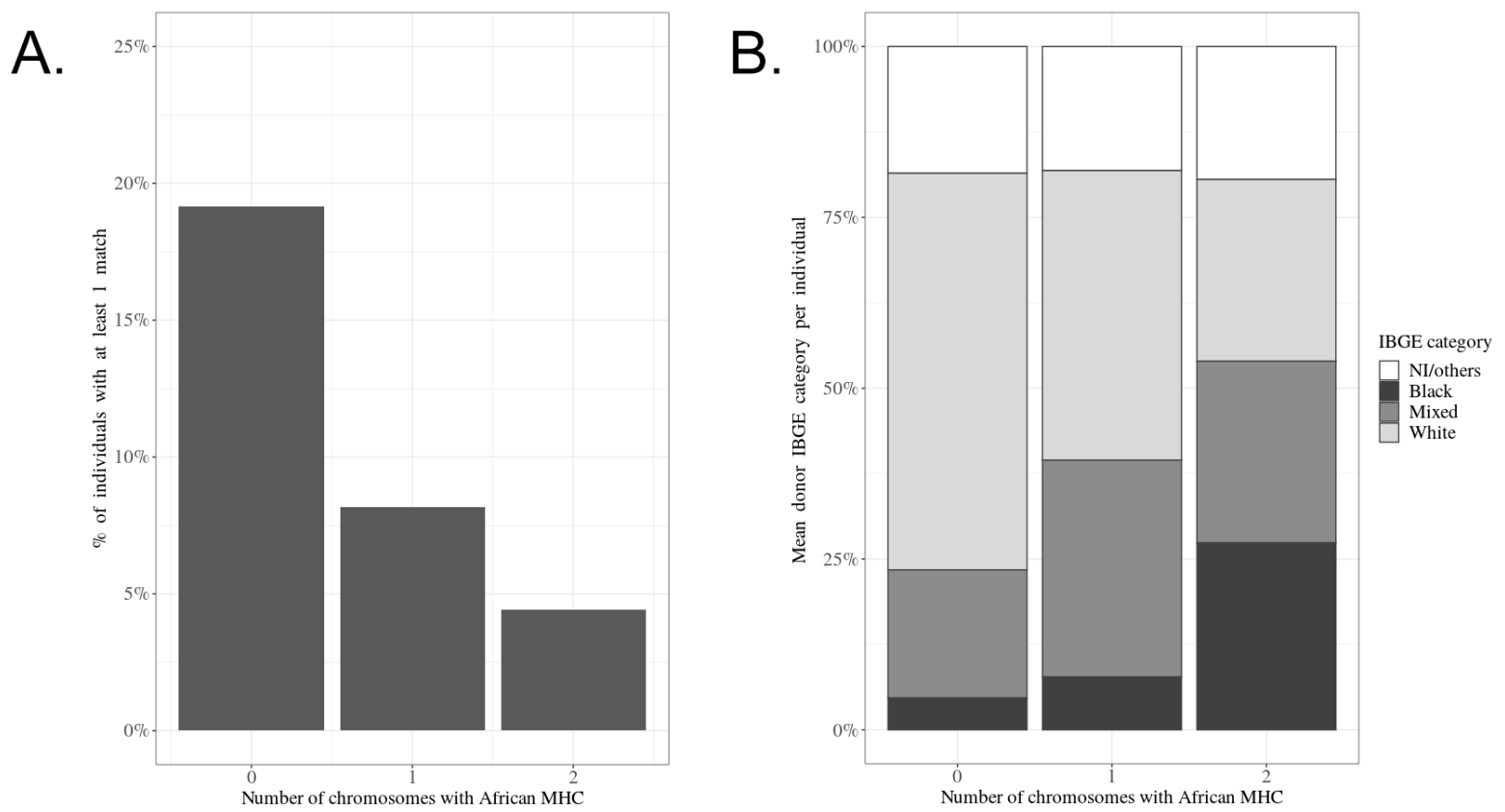

Figure 5. Ancestry and donor availability in the Brazilian bone marrow registry (for matching at both alleles at 5 loci, or 10/10 matching). Admixed individuals were classified regarding the genetic ancestry of their MHC region, using RFMIX ("RFMix: A Discriminative Modeling Approach for Rapid and Robust Local-Ancestry Inference" 2013). Each individual was assigned a value, referring to the number of chromosomes for which the $\mathrm{MHC}$ region was African: 0,1 , or 2. (A) Effect of African ancestry in the MHC on the proportion of individuals that find at least one match in the registry. The y axis represents the proportions of individuals who found at least one compatible donor. (B) The self-assigned identifier (a proxy coarsely related to ancestry) of potential donors, with respect to the genetic ancestry in the MHC region. Notice that for individuals with 2 African chromosomes in the MHC region, "Black" and "Mixed" make up the largest fraction of potential donors. (Adapted from Nunes et al., 2020).

\section{Conclusion}

The analysis of population structure at HLA connects with themes that Richard Lewontin explored in his scientific work and his communication about science to the non-specialized audience.

Lewontin's writings on evolutionary biology recurrently emphasized the challenge of delimiting units of analysis, both at the morphological and genetic levels (Lewontin 2000)Lewontin, 2000). Indeed, there is no single correct way to define genotypes for the 
population structure analyses. We have shown that results for the diversity of SNPs and HLA alleles are both informative, but convey different types of information. The SNP level analysis captures the fact that most nucleotide level variation is widely shared among populations and regions (as in fact they are shared among species, (Leffler et al. 2013). However, such extensive sharing at the SNP level does not translate to sharing of HLA alleles among populations and regions, with the repertoire of HLA alleles differing markedly among regions (Figure 4). The decision with respect to which definition of "HLA genotype" will be used has a bearing on the distribution of genetic variation.

Lewontin's work also emphasized the context-dependency of evolutionary trajectories, with empirical and analytical explorations of fitness surfaces that arise when interactions among loci and changing environments are considered (Lewontin and White 1960). The study of HLA genes provides an example of how a locus can carry the signatures of distinct selective regimes, at different timescales: strong evidence of balancing selection at the level of SNPs, when long timescales are considered, and evidence of recent selection, favoring locally adapted HLA alleles, at recent timescale (involving divergence between populations inhabiting the same continent, or recently admixed populations).

Finally, Lewontin was explicit about his political views and assumed that scientists' technical work reflected their inevitable (although not always stated) political perspectives. The study of HLA population structure reveals how a technical question, regarding the degree of population structure in a genomic region, can have a bearing on public health issues in admixed populations. We ourselves were stimulated to investigate the effects of ancestry on the chances of finding donors by both recent scientific work in the United States (Gragert et al. 2014), and by websites maintained by patient-driven organizations (for example,

https://blackbonemarrow.com/ and https://bonemarrowwish.org/). In the case of Brazil, the long history of slavery lies at the root of a pattern of systemic racism (Ribeiro 2019), and has resulted in a social organization where individuals of African ancestry have reduced access to quality healthcare, and are overburdened by many diseases, including COVID-19 (Martins-Filho et al. 2021). This calls for, among other efforts, research with the potential to guide institutional health care strategies. In the specific case of the Brazilian registry, strategies to recruit donors of African ancestry are a direct recommendation of the genetic analyses. 


\section{Acknowledgements}

We thank the organizers of this special issue for their invitation to contribute. DM thanks the members of the Evolutionary Genetics Laboratory at the University of São Paulo for helpful discussions.

\section{Funding}

This work was supported by the United States National Institutes of Health - NIH (R01 GM075091; KN, BSW, DM), and São Paulo Research Foundation - FAPESP grant 2019/11593-9 (to ASM).

\section{Authors' contributions}

Conceptualization (DM)

Data curation (KN, ASM, EC, NSBS)

Formal analysis (KN, ASM, DM, JG, BSW, NSBS, EC)

Funding acquisition (BSW, DM)

Investigation (KN, ASM, DM, BSW, JG)

Methodology (EC)

Supervision (DM, JG, BSW)

Writing - original draft (DM)

Writing - review \& editing (DM with input from all authors) 


\section{References}

1000 Genomes Project Consortium, and Adam Auton, Lisa D Brooks, Richard M Durbin, Erik P Garrison, Hyun Min Kang, Jan O Korbel, Jonathan L Marchini, Shane McCarthy, Gil A McVean, Gonçalo R Abecasis. 2015. "A Global Reference for Human Genetic Variation." Nature 526 (7571): 68-74.

Ade Ajayi, J. F. 1998. UNESCO General History of Africa, Vol. VI, Abridged Edition: Africa in the Nineteenth Century Until the 1880s. University of California Press.

Balloux, F., H. Brünner, N. Lugon-Moulin, J. Hausser, and J. Goudet. 2000. "Microsatellites Can Be Misleading: An Empirical and Simulation Study." Evolution; International Journal of Organic Evolution 54 (4): 1414-22.

Bhatia, Gaurav, Nick Patterson, Bogdan Pasaniuc, Noah Zaitlen, Giulio Genovese, Samuela Pollack, Swapan Mallick, et al. 2011. "Genome-Wide Comparison of African-Ancestry Populations from CARe and Other Cohorts Reveals Signals of Natural Selection." American Journal of Human Genetics 89 (3): 368-81.

Bitarello, Bárbara D., Cesare de Filippo, João C. Teixeira, Joshua M. Schmidt, Philip Kleinert, Diogo Meyer, and Aida M. Andrés. 2018. "Signatures of Long-Term Balancing Selection in Human Genomes." Genome Biology and Evolution 10 (3): 939-55.

Bodmer, W. F. 1972. "Evolutionary Significance of the HL-A System." Nature 237 (5351): 139-45 passim.

Brandt, Débora Y. C., Vitor R. C. Aguiar, Bárbara D. Bitarello, Kelly Nunes, Jérôme Goudet, and Diogo Meyer. 2015. "Mapping Bias Overestimates Reference Allele Frequencies at the HLA Genes in the 1000 Genomes Project Phase I Data." G3 5 (5): 931-41.

Brandt, Débora Y. C., Jônatas César, Jérôme Goudet, and Diogo Meyer. 2018. "The Effect of Balancing Selection on Population Differentiation: A Study with HLA Genes." G3 8 (8): 2805-15.

Buhler, Stéphane, and Alicia Sanchez-Mazas. 2011. "HLA DNA Sequence Variation among Human Populations: Molecular Signatures of Demographic and Selective Events." PLoS ONE. https://doi.org/10.1371/journal.pone.0014643.

Byrska-Bishop, Marta, Uday S. Evani, Xuefang Zhao, Anna O. Basile, Haley J. Abel, Allison A. Regier, André Corvelo, et al. 2021. "High Coverage Whole Genome Sequencing of the Expanded 1000 Genomes Project Cohort Including 602 Trios." Biorxiv. https://doi.org/10.1101/2021.02.06.430068.

Castelli, Erick C., Michelle A. Paz, Andréia S. Souza, Jaqueline Ramalho, and Celso Teixeira Mendes-Junior. 2018. "Hla-Mapper: An Application to Optimize the Mapping of HLA Sequences Produced by Massively Parallel Sequencing Procedures." Human Immunology 79 (9): 678-84.

Chang, Christopher C., Carson C. Chow, Laurent Cam Tellier, Shashaank Vattikuti, Shaun M. Purcell, and James J. Lee. 2015. "Second-Generation PLINK: Rising to the Challenge of Larger and Richer Datasets." GigaScience 4 (1). https://doi.org/10.1186/s13742-015-0047-8.

Danecek, Petr, Adam Auton, Goncalo Abecasis, Cornelis A. Albers, Eric Banks, Mark A. DePristo, Robert E. Handsaker, et al. 2011. "The Variant Call Format and VCFtools." Bioinformatics 27 (15): 2156-58.

Garrigan, Daniel, and Philip W. Hedrick. 2003. "Perspective: Detecting Adaptive Molecular Polymorphism: Lessons from the MHC." Evolution; International Journal of Organic Evolution 57 (8): 1707-22.

Goudet, Jerome. 2005. "Hierfstat, a Package for R to Compute and Test Hierarchical F-Statistics." Molecular Ecology Notes. https://doi.org/10.1111/j.1471-8286.2004.00828.x. 
Gragert, Loren, Mary Eapen, Eric Williams, John Freeman, Stephen Spellman, Robert Baitty, Robert Hartzman, et al. 2014. "HLA Match Likelihoods for Hematopoietic Stem-Cell Grafts in the U.S. Registry." The New England Journal of Medicine 371 (4): 339-48.

Hedrick, Philip W. 2005. "A Standardized Genetic Differentiation Measure." Evolution 59 (8): 1633-38.

Hedrick, Philip W., and Glenys Thomson. 1983. "EVIDENCE FOR BALANCING SELECTION AT HLA." Genetics. https://doi.org/10.1093/genetics/104.3.449.

Hughes, A. L., and M. Nei. 1992. "Maintenance of MHC Polymorphism." Nature.

Jost, Lou. 2008. "GSTand Its Relatives Do Not Measure Differentiation." Molecular Ecology. https://doi.org/10.1111/j.1365-294x.2008.03887.x.

Klein, Jan, and Akie Sato. 2000. "The HLA System." The New England Journal of Medicine 343 (10): 702-9.

Leffler, Ellen M., Ziyue Gao, Susanne Pfeifer, Laure Ségurel, Adam Auton, Oliver Venn, Rory Bowden, et al. 2013. "Multiple Instances of Ancient Balancing Selection Shared between Humans and Chimpanzees." Science 339 (6127): 1578-82.

Lewontin, R. C. 2000. The Triple Helix: Gene, Organism and Environment. Vol. 6. Harvard University Press.

Lewontin, R. C., and J. Krakauer. 1973. "Distribution of Gene Frequency as a Test of the Theory of the Selective Neutrality of Polymorphisms." Genetics 74 (1): 175-95.

Lewontin, R. C., and M. J. D. White. 1960. "Interaction between Inversion Polymorphisms of Two Chromosome Pairs in the Grasshopper Moraba Scurra." Evolution 14 (1): 116-29.

Manica, Andrea, Franck Prugnolle, and François Balloux. 2005. "Geography Is a Better Determinant of Human Genetic Differentiation than Ethnicity." Human Genetics 118 (3-4): 366-71.

Martins-Filho, Paulo Ricardo, Brenda Carla Lima Araújo, Karyna Batista Sposato, Adriano Antunes de Souza Araújo, Lucindo José Quintans-Júnior, and Victor Santana Santos. 2021. "Racial Disparities in COVID-19-Related Deaths in Brazil: Black Lives Matter?" Journal of Epidemiology 31 (3): 239.

Meyer, Diogo, Vitor R. C. Aguiar, Bárbara D. Bitarello, Débora Y. C. Brandt, and Kelly Nunes. 2018. "A Genomic Perspective on HLA Evolution." Immunogenetics. https://doi.org/10.1007/s00251-017-1017-3.

Meyer, Diogo, Richard M. Single, Steven J. Mack, Henry A. Erlich, and Glenys Thomson. 2006. "Signatures of Demographic History and Natural Selection in the Human Major Histocompatibility Complex Loci." Genetics 173 (4): 2121-42.

Nei, Masatoshi, and Takeo Maruyama. 1975. "Lewontin-Krakauer Test for Neutral Genes." Genetics. https://doi.org/10.1093/genetics/80.2.395.

Nielsen, Rasmus. 2005. "Molecular Signatures of Natural Selection." Annual Review of Genetics. https://doi.org/10.1146/annurev.genet.39.073003.112420.

Norris, Emily T., Lavanya Rishishwar, Aroon T. Chande, Andrew B. Conley, Kaixiong Ye, Augusto Valderrama-Aguirre, and I. King Jordan. 2021. "Admixture-Enabled Selection for Rapid Adaptive Evolution in the Americas." Genome Biology 21 (29): 1-12.

Nunes, Kelly, Maria Helena Thomaz Maia, Eduardo José Melo Dos Santos, Sidney Emanuel Batista Dos Santos, João Farias Guerreiro, Maria Luiza Petzl-Erler, Gabriel Bedoya, et al. 2021. "How Natural Selection Shapes Genetic Differentiation in the MHC Region: A Case Study with Native Americans." Human Immunology 82 (7): 523-31.

Ochoa, Alejandro, and John D. Storey. 2021. "Estimating FST and Kinship for Arbitrary Population Structures." PLoS Genetics 17 (1): e1009241.

Patin, Etienne, Marie Lopez, Rebecca Grollemund, Paul Verdu, Christine Harmant, Hélène Quach, Guillaume Laval, et al. 2017. "Dispersals and Genetic Adaptation of Bantu-Speaking Populations in Africa and North America." Science 356 (6337): 543-46. Prugnolle, Franck, Andrea Manica, and François Balloux. 2005. "Geography Predicts Neutral 
Genetic Diversity of Human Populations." Current Biology: CB 15 (5): R159-60.

"RFMix: A Discriminative Modeling Approach for Rapid and Robust Local-Ancestry Inference." 2013. American Journal of Human Genetics 93 (2): 278-88.

Ribeiro, Djamila. 2019. Pequeno Manual Antirracista.

Rosenberg, Noah A., Saurabh Mahajan, Sohini Ramachandran, Chengfeng Zhao, Jonathan K. Pritchard, and Marcus W. Feldman. 2005. "Clines, Clusters, and the Effect of Study Design on the Inference of Human Population Structure." PLoS Genetics 1 (6): e70.

Salzano, Francisco M., and Maria C. Bortolini. 2005. The Evolution and Genetics of Latin American Populations. Cambridge University Press.

Sanchez-Mazas, Alicia. 2007. "An Apportionment of Human HLA Diversity." Tissue Antigens 69 Suppl 1 (April): 198-202.

Solberg, O. D., Mack, S. J., Lancaster, A. K., Single, R. M., Tsai, Y., Sanchez-Mazas, A., \& Thomson, G. 2008. "Balancing Selection and Heterogeneity across the Classical Human Leukocyte Antigen Loci: A Meta-Analytic Review of 497 Population Studies." Human Immunology 69 (7): 443-64.

Souza, Aracele Maria de, Sarah Stela Resende, Taís Nóbrega de Sousa, and Cristiana Ferreira Alves de Brito. 2019. "A Systematic Scoping Review of the Genetic Ancestry of the Brazilian Population." Genetics and Molecular Biology 42 (3): 495-508.

Weir, Bruce S., Lon R. Cardon, Amy D. Anderson, Dahlia M. Nielsen, and William G. Hill. 2005. "Measures of Human Population Structure Show Heterogeneity among Genomic Regions." Genome Research 15 (11): 1468-76.

Weir, Bruce S., and Jérôme Goudet. 2017. "A Unified Characterization of Population Structure and Relatedness." Genetics 206 (4): 2085-2103.

Weir, B. S., and C. Clark Cockerham. 1984. "Estimating F-Statistics for the Analysis of Population Structure." Evolution 38 (6): 1358-70.

Weir, B. S., and W. G. Hill. 2002. "Estimating F-Statistics." Annual Review of Genetics 36 (June): 721-50.

Zhou, Quan, Liang Zhao, and Yongtao Guan. 2016. "Strong Selection at MHC in Mexicans since Admixture." PLOS Genetics. https://doi.org/10.1371/journal.pgen.1005847. 